\title{
Retrospective study of biopsied head and neck lesions in a cohort of referral Taiwanese patients
}

Frank Lei ${ }^{1 \dagger}$, Ping-Ho Chen ${ }^{2}$, Jing-Yi Chen ${ }^{3}$, Wen-Chen Wang ${ }^{2,3}$, Li-Min Lin ${ }^{2,3,4}$, Hsien-Cheng Huang ${ }^{3}$, Kun-Yen Ho ${ }^{1,2 \dagger}$, Chung-Ho Chen ${ }^{5}$ and Yuk-Kwan Chen $2,3,4^{*}$

\begin{abstract}
Introduction: A study of the whole spectrum of biopsied head and neck (HN) diseases in Taiwan has not yet been performed. Therefore, the current study aimed to provide updated information about HN lesions in a cohort of referral Taiwanese patients for histopathological examination.

Methods: HN lesions (2000-2011) in patients with records of age, sex, and histological diagnoses were retrieved from the Oral Pathology Department of the institution. These lesions were classified into four main categories:

tumor/tumor-like reactive lesions, cystic/pseudocystic lesions, inflammatory/infective lesions, and others/ miscellaneous lesions.

Results: A total of $37,210 \mathrm{HN}$ lesions were included in the current study. Most of these lesions were distributed in the group of tumor/tumor-like reactive lesions, followed by the groups of inflammatory/infective lesions, cystic/ pseudocystic lesions, and others/miscellaneous lesions. Squamous cell carcinoma was the most common HN lesion, and was also the most frequent malignant lesion among the referral patients.
\end{abstract}

Conclusion: It was worthy of note that squamous cell carcinoma and oral potentially malignant disorders comprised high percentages of all HN lesions for the present cohort of referral patients.

Keywords: Oral lesions, Oral health

\section{Introduction}

Reviewing the English literature, to our knowledge, most of the previous studies of $\mathrm{HN}$ lesions analyzed specific diseases, such as odontogenic cysts or tumors [1,2], in certain populations, such as pediatric or geriatric populations [3,4]. There are only a few retrospective reports focusing on the prevalence the whole spectrum of biopsied oral and maxillofacial (OMF) lesions in various countries [5-11]. Information of the types and frequency of $\mathrm{HN}$ lesions in the local population may always be helpful in management the patients. A study of a variety of biopsied HN diseases in Taiwanese patients has not yet been performed. Hence, the present study aimed to provide updated information about $\mathrm{HN}$ lesions in a

\footnotetext{
* Correspondence: k840042@gmail.com

${ }^{\dagger}$ Equal contributors

${ }^{2}$ School of Dentistry, College of Dental Medicine, Kaohsiung Medical

University, Kaohsiung, Taiwan

${ }^{3}$ Division of Oral Pathology \& Maxillofacial Radiology, Kaohsiung Medical

University Hospital, Kaohsiung, Taiwan

Full list of author information is available at the end of the article
}

cohort of referral Taiwanese patients for histopathological examination.

\section{Materials and methods}

The Oral Pathology Department of the institution is the department providing a histopathological service encompassed by HN surgery specialty in southern Taiwan, receiving specimens mainly from the surgeons of the OMF Surgery Department, ENT Department, and Plastic Surgery Department of the hospital as well as other nearby regional hospitals and local dental clinics. Three experienced board-certified HN pathologists make the histological diagnosis for each biopsy independently, based mostly on paraffin embedded sections of hematoxylineosin staining and sometimes conjunction with immunohistochemical and/or histochemical staining. The histological diagnoses are established by peer slide review; however, if disagreement exists amongst the pathologists, a consensus is reached upon mutual discussion. 
This study complied with the Helsinki Declaration with the data collected after the approval of the Institutional Review Board of the hospital (KMUH-IRB-2014-73). A total of 39,503 diagnosed cases in the $\mathrm{HN}$ region between 2000 and 2011 were retrieved from the database of the Oral Pathology Department. With the exclusion of normal tissues and lesions without specific findings, a pool of 37,210 cases was included for analyses. Age, sex, and histological diagnoses were recorded for these HN lesions, which were classified into four main categories: tumor/ tumor-like reactive lesions, cystic/pseudocystic lesions, inflammatory/infective lesions, and others/miscellaneous lesions.

Statistical analyses (chi-square test/binominal proportion test) for prevalence rates of the lesions, age and sex distributions of the patients were performed using SAS Statistical Package (Version 9.1.3, SAS Institute Inc., Cary, NC, USA). Since the number of the items of some tables was as large as approximately 50, we employed the Bonferroni method (threshold of $\mathrm{p}=0.001 ; \mathrm{p}_{0} / \mathrm{N}$, $\mathrm{p}_{0}=0.05, \mathrm{~N}=50$ items) for multiple testing-adjusted corrections. Hence, the results were considered significant when the $\mathrm{p}$ value was $<0.001$ (i.e. $0.05 / 50$ ). If $\mathrm{p}<0.0001$, the research findings were very highly significant.

\section{Results}

The frequencies of the 12 most common HN lesions, with a total number of 28,783 , comprised $77.3 \%$ of all the lesions in the current study, are shown in Table 1 . The most common disease in the present cohort was squamous cell carcinoma (SCC, 13.3\%), followed by hyperkeratosis (HK, $12.8 \%)$, epithelial dysplasia (ED, 7.8\%), candidiasis (6.8\%), oral submucous fibrosis (OSF, 6.7\%) and epithelial hyperplasia (EH, 6.4\%); the aforementioned first six

Table 1 Number and percentages of the 12 most common head \& neck lesions

\begin{tabular}{lcc}
\hline $\mathbf{1 2}$ most common lesions & Number & \% of all lesions \\
\hline Squamous cell carcinoma & 4960 & 13.3 \\
Hyperkeratosis* & 4779 & 12.8 \\
Epithelial dysplasia* & 2899 & 7.8 \\
Candidiasis & 2535 & 6.8 \\
Oral submucous fibrosis* & 2500 & 6.7 \\
Epithelial hyperplasia* & 2371 & 6.4 \\
Verrucous hyperplasia* & 1850 & 5.0 \\
Inflammation & 1821 & 4.9 \\
Radicular cyst & 1720 & 4.6 \\
Apical granuloma & 1395 & 3.8 \\
Non-specific ulcer & 986 & 2.6 \\
Mucocele & 967 & 2.6 \\
Total number & 28783 & 77.3 \\
\hline
\end{tabular}

*Oral potentially malignant disorders. most common lesions constituted more than $50 \%$ of all the $\mathrm{HN}$ lesions. With the exception of $\mathrm{HK}$, the percentage of SCC was significant higher than ED, candidiasis, OSF, and EH ( $\mathrm{p}<0.0001)$.

Various groups of HN lesions are classified in Table 2. The highest number of lesions were distributed in the group of oral potentially malignant disorders (38.7\%), followed by the groups of inflammatory/infective lesions (31.6\%) and non-odontogenic malignant lesions (16.2\%). In contrast, the lowest number of lesions was found in the non-odontogenic cystic/pseudocystic group, in which only 91 cases were included. The percentage of oral potentially malignant disorders was significant higher than inflammatory/infective lesions and non-odontogenic malignant lesions $(\mathrm{p}<0.0001)$. Also, the percentage of inflammatory/ infective lesions was significant higher than and nonodontogenic malignant lesions ( $\mathrm{p}<0.0001)$.

The sex and age distribution of the HN lesion patients is shown in Table 3. Most lesions were distributed in the range of 50-59 years, followed by 40-49 years, and 30-39 years, all of which comprised more than $70.0 \%$ of all $\mathrm{HN}$ lesions. The percentage of the patients in the range of 50-59 years was significantly higher than the ranges of 40-49 years and 30-39 year respectively $(\mathrm{p}<0.0001)$ whilst the percentage of the range of 40-49 year was

Table 2 Number and percentages of the four categories of head \& neck lesions

\begin{tabular}{|c|c|c|c|}
\hline Categories & & Number & $\begin{array}{l}\% \text { of } \\
\text { total }\end{array}$ \\
\hline \multirow{13}{*}{$\begin{array}{l}\text { Tumor/tumor-like } \\
\text { reactive lesions }\end{array}$} & Odontogenic (benign) & 456 & 1.23 \\
\hline & Non-odontogenic (benign) & & \\
\hline & Bone & 160 & 0.43 \\
\hline & Salivary gland & 96 & 0.26 \\
\hline & Epithelial & 118 & 0.32 \\
\hline & Soft tissue & 1251 & 3.36 \\
\hline & $\begin{array}{l}\text { Non-odontogenic (oral } \\
\text { potentially malignant disorder) }\end{array}$ & & \\
\hline & Epithelial & 14399 & 38.70 \\
\hline & Non-odontogenic (malignant) & & \\
\hline & Mesenchymal & 15 & 0.04 \\
\hline & Hematologic & 45 & 0.12 \\
\hline & Salivary gland & 82 & 0.22 \\
\hline & Epithelial & 5886 & 15.82 \\
\hline \multirow{2}{*}{$\begin{array}{l}\text { Cystic/pseudocystic } \\
\text { lesions }\end{array}$} & Odontogenic & 2274 & 6.11 \\
\hline & Non-odontogenic & 91 & 0.25 \\
\hline $\begin{array}{l}\text { Inflammatory/ } \\
\text { infective lesions }\end{array}$ & & 11775 & 31.65 \\
\hline $\begin{array}{l}\text { Others/miscellanous } \\
\text { lesions }\end{array}$ & & 562 & 1.51 \\
\hline
\end{tabular}


Table 3 Age and sex distribution of the patients presenting head \& neck lesions

\begin{tabular}{lcc}
\hline Age (years) & Number & Male:Female \\
\hline $0-9$ & 362 & $1.1: 1$ \\
$10-19$ & 970 & $1.2: 1$ \\
$20-29$ & 2505 & $1.6: 1$ \\
$30-39$ & 5733 & $4.2: 1$ \\
$40-49$ & 9871 & $5.5: 1$ \\
$50-59$ & 11053 & $4.4: 1$ \\
$60-69$ & 3400 & $3.1: 1$ \\
$70-79$ & 2676 & $1.6: 1$ \\
$80-89$ & 608 & $0.8: 1$ \\
$90-99$ & 32 & $0.3: 1$ \\
Total & $\mathbf{3 7 2 1 0}$ & $\mathbf{3 . 4 : 1}$ \\
\hline
\end{tabular}

significant higher than the range of $30-39$ year $(\mathrm{p}<0.0001)$. Moreover, in the above three age groups, the number of male patients was higher significantly than the number of female patients, particularly in the highest male to female ratio in the range of $40-49$ years $(\mathrm{p}<0.0001)$, whereas the lowest male to female ratio was found in the range of $90-99$ years $(\mathrm{p}=0.0023)$. The lowest number of lesions was found in the range of 90-99 years.

The data of the odontogenic cyst group are presented in Table 4, with a male to female ratio of about 1.3:1. $(\mathrm{p}<0.0001)$. Radicular cyst was the most common lesion, comprising about $75.0 \%$ of the lesions within this group, and was mostly distributed in the third to the sixth decades of life significantly higher than other groups ( $\mathrm{p}<0.0001)$. The second and the third most common lesions in this group were dentigerous cyst and keratocystic odontogenic tumor (formerly called odontogenic keratocyst), respectively; both lesions collectively comprised about $20.0 \%$ of the lesions. A significant difference of distribution percentage was found among radicular cyst, dentigerous cyst and keratocystic odontogenic tumor $(\mathrm{p}<0.0001)$.

Only 91 cases were contained in the group of nonodontogenic cystic/pseudocystic lesions (Table 5), with a significant difference of male to female ratio of $2.8: 1$ $(\mathrm{p}<0.0001)$. The most common lesion in this group was epidermoid cyst, followed by lymphoepithelial cyst and nasopalatine duct cyst. The percentage of epidermoid cyst was significant higher than lymphoepithelial cyst and nasopalatine duct cyst $(\mathrm{p}<0.0001)$ whilst the percentage of lymphoepithelial cyst and nasopalatine duct cyst was similar to each other $(p<0.4328)$. Most of the epidermoid cysts were distributed in the range of 40-49 years, and the number of male patients was much higher than that of female patients $(\mathrm{p}<0.0001)$; the second (lymphoepithelial cyst) and the third (nasopalatine duct cyst) most common lesions were mostly found in the first four decades of life.

The data of benign odontogenic tumors are listed in Table 6, with a male to female ratio of almost 1:1 $(\mathrm{p}=0.9254)$. The most common lesion within this group was odontoma, comprising about $40.0 \%$ of the lesions, which was predominantly found in the range of 10-19 years compared with the other groups $(\mathrm{p}<0.0001)$. The second most common lesion was ameloblastoma, which was mostly distributed in the second and the third decade of life. There were no significant differences between odontoma and ameloblastoma ( $\mathrm{p}=0.0557)$.

Most of the HN lesions in the current study were distributed in the group of benign non-odontogenic tumor/ tumor-like reactive lesions (Table 7). The most common lesion of this group was noted in the epithelial subgroup, followed by the soft tissue subgroup, bone subgroup, and salivary gland subgroup. The percentage of the epithelial subgroup ( $n=14,517)$ was significantly higher than the soft tissue subgroup $(n=1,251)$, bone subgroup $(n=160)$, and salivary gland subgroup $(\mathrm{n}=96)(\mathrm{p}<0.0001)$. Also, the

Table 4 Number, sex, and age distribution of the patients presenting odontogenic cysts

\begin{tabular}{|c|c|c|c|c|c|c|c|c|c|c|c|c|}
\hline \multirow{2}{*}{$\begin{array}{l}\text { Odontogenic cysts/ } \\
\text { pesudocysts }\end{array}$} & \multirow[t]{2}{*}{ Male } & \multirow[t]{2}{*}{ Female } & \multicolumn{10}{|c|}{ Age distribution (years) } \\
\hline & & & $0-9$ & 10-19 & $20-29$ & $30-39$ & $40-49$ & $50-59$ & $60-69$ & $70-79$ & $80-89$ & $90-99$ \\
\hline Radicular cyst & 910 & 810 & 15 & 98 & 313 & 343 & 368 & 374 & 79 & 105 & 25 & 0 \\
\hline Dentigerous cyst & 200 & 98 & 28 & 65 & 54 & 39 & 53 & 38 & 8 & 11 & 2 & 0 \\
\hline Keratocystic odontogenic tumor & 106 & 64 & 4 & 40 & 46 & 34 & 25 & 14 & 2 & 3 & 2 & 0 \\
\hline Residual cyst & 28 & 20 & 2 & 3 & 1 & 10 & 3 & 17 & 2 & 10 & 0 & 0 \\
\hline Calcifying odontogenic cyst & 13 & 5 & 1 & 7 & 3 & 2 & 3 & 0 & 1 & 1 & 0 & 0 \\
\hline Paradental cyst & 4 & 3 & 0 & 0 & 4 & 2 & 1 & 0 & 0 & 0 & 0 & 0 \\
\hline Glandular odontogenic cyst & 4 & 0 & 0 & 0 & 0 & 1 & 1 & 0 & 2 & 0 & 0 & 0 \\
\hline Eruption cyst & 4 & 0 & 1 & 3 & 0 & 0 & 0 & 0 & 0 & 0 & 0 & 0 \\
\hline Lateral periodontal cyst & 2 & 2 & 0 & 0 & 1 & 0 & 0 & 1 & 2 & 0 & 0 & 0 \\
\hline Gingival cyst & 1 & 0 & 1 & 0 & 0 & 0 & 0 & 0 & 0 & 0 & 0 & 0 \\
\hline Total & 1272 & 1002 & 52 & 216 & 422 & 431 & 454 & 444 & 96 & 130 & 29 & 0 \\
\hline
\end{tabular}


Table 5 Number, sex, and age distribution of the patients presenting non-odontogenic cyst/pesudocysts

\begin{tabular}{|c|c|c|c|c|c|c|c|c|c|c|c|c|}
\hline \multirow{2}{*}{$\begin{array}{l}\text { Non-odontogenic } \\
\text { cyst/pesudocysts }\end{array}$} & \multirow[t]{2}{*}{ Male } & \multirow[t]{2}{*}{ Female } & \multicolumn{10}{|c|}{ Age distribution (years) } \\
\hline & & & $0-9$ & 10-19 & $20-29$ & $30-39$ & $40-49$ & $50-59$ & $60-69$ & $70-79$ & $80-89$ & $90-99$ \\
\hline Epidermoid cyst & 34 & 7 & 0 & 2 & 4 & 6 & 16 & 6 & 3 & 3 & 1 & 0 \\
\hline Lymphoepithelial cyst & 9 & 6 & 0 & 1 & 2 & 4 & 3 & 3 & 1 & 1 & 0 & 0 \\
\hline Nasopalatine duct cyst & 7 & 4 & 0 & 0 & 2 & 2 & 1 & 4 & 2 & 0 & 0 & 0 \\
\hline Simple bone cyst & 2 & 3 & 0 & 3 & 0 & 1 & 1 & 0 & 0 & 0 & 0 & 0 \\
\hline Aneurysmal bone cyst & 4 & 0 & 0 & 0 & 3 & 0 & 0 & 1 & 0 & 0 & 0 & 0 \\
\hline Sebaceous cyst & 2 & 1 & 0 & 0 & 2 & 0 & 1 & 0 & 0 & 0 & 0 & 0 \\
\hline Nasolabial cyst & 2 & 1 & 0 & 0 & 0 & 0 & 1 & 2 & 0 & 0 & 0 & 0 \\
\hline Thyroglossal duct cyst & 3 & 0 & 0 & 0 & 0 & 0 & 2 & 0 & 1 & 0 & 0 & 0 \\
\hline Incisive canal cyst & 2 & 2 & 0 & 0 & 3 & 1 & 0 & 0 & 0 & 0 & 0 & 0 \\
\hline Branchial cleft cyst & 1 & 0 & 0 & 0 & 0 & 0 & 0 & 0 & 0 & 1 & 0 & 0 \\
\hline Globulomaxillary cyst & 1 & 0 & 0 & 0 & 0 & 1 & 0 & 0 & 0 & 0 & 0 & 0 \\
\hline Total & 67 & 24 & 0 & 6 & 16 & 15 & 25 & 16 & 7 & 5 & 1 & 0 \\
\hline
\end{tabular}

percentage of the soft tissue subgroup $(n=1251)$ was significantly higher than the bone subgroup $(n=160)$, and salivary gland subgroup $(\mathrm{n}=96)(\mathrm{p}<0.0001)$. The percentage of the bone subgroup $(n=160)$ was significantly higher than the salivary gland subgroup $(\mathrm{n}=96)$ $(\mathrm{p}<0.0001)$.

Five of the seven types of lesions in the epithelial subgroup were potentially malignant disorders, and all these five lesions belonged to the top 12 diseases. The most common lesion of the potentially malignant disorders was HK, followed by ED and OSF. The number of male patients was almost ten times that of the female patients in all the lesions of potentially malignant disorders $(\mathrm{p}<0.0001)$.

Fibroma, comprising about $50 \%$ of the lesions, was the most common lesion in the soft tissue subgroup, significantly higher than the other groups $(p<0.0001)$, with the number of male patients almost equal to that of female patients $(\mathrm{p}=0.9045)$. Hemangioma was the second most common lesion, which was mostly found in the sixth decade of life. In the hemangioma, the percentage of sixth decade of life was observed no significantly difference than the age $40-49(\mathrm{p}=0.0550)$. The $50-59$ age group compared with other groups, the $\mathrm{p}$ value was $<0.0001$, except for 30-39 age group $(p=0.0080)$, and $60-69$ age group ( $\mathrm{p}=0.0001)$.

Cemento-ossifying fibroma, cemento-osseous dysplasia, and fibrous dysplasia, comprising about $76.0 \%$ of the lesions, were the three most common lesions within the bone subgroup, in which the number of males was lower than that of females. The percentage of cementoossifying fibroma was observed higher than cementoosseous dysplasia $(\mathrm{p}=0.0171)$. Compared with fibrous dysplasia, cemento-ossifying fibroma had higher percentage than fibrous dysplasia $(p=0.0019)$. There were no difference of percentage between cemento-osseous

Table 6 Number, sex, and age distribution of the patients presenting benign odontogenic tumors

\begin{tabular}{|c|c|c|c|c|c|c|c|c|c|c|c|c|}
\hline \multirow[t]{2}{*}{ Odontogenic (benign) tumors } & \multirow[t]{2}{*}{ Male } & \multirow[t]{2}{*}{ Female } & \multicolumn{10}{|c|}{ Age distribution (years) } \\
\hline & & & $0-9$ & 10-19 & $20-29$ & $30-39$ & $40-49$ & $50-59$ & $60-69$ & $70-79$ & $80-89$ & $90-99$ \\
\hline Odontoma & 92 & 103 & 37 & 94 & 29 & 14 & 7 & 12 & 2 & 0 & 0 & 0 \\
\hline Ameloblastoma & 92 & 67 & 5 & 14 & 42 & 35 & 24 & 27 & 11 & 1 & 0 & 0 \\
\hline Odontogenic fibroma & 17 & 29 & 4 & 8 & 12 & 9 & 5 & 4 & 2 & 2 & 0 & 0 \\
\hline Odontogenic myxoma & 11 & 9 & 0 & 8 & 5 & 1 & 4 & 0 & 0 & 2 & 0 & 0 \\
\hline Cementoblastoma & 3 & 6 & 0 & 1 & 0 & 2 & 3 & 1 & 1 & 0 & 1 & 0 \\
\hline Adenomatoid odontogenic tumor & 2 & 6 & 0 & 4 & 0 & 2 & 2 & 0 & 0 & 0 & 0 & 0 \\
\hline Ameloblastic odontoma & 6 & 2 & 2 & 6 & 0 & 0 & 0 & 0 & 0 & 0 & 0 & 0 \\
\hline Calcifying epithelial odontogenic tumor & 0 & 5 & 0 & 0 & 1 & 0 & 4 & 0 & 0 & 0 & 0 & 0 \\
\hline Ameloblastic fibroma & 3 & 1 & 3 & 1 & 0 & 0 & 0 & 0 & 0 & 0 & 0 & 0 \\
\hline Squamous odontogenic tumor & 1 & 1 & 1 & 0 & 0 & 0 & 0 & 0 & 1 & 0 & 0 & 0 \\
\hline Total & 227 & 229 & 52 & 136 & 89 & 63 & 49 & 44 & 17 & 5 & 1 & 0 \\
\hline
\end{tabular}


Table 7 Number, sex and age distribution of patients presenting benign non-odontogenic tumor/tumor-like reactive lesions

\begin{tabular}{|c|c|c|c|c|c|c|c|c|c|c|c|c|}
\hline \multirow{2}{*}{$\begin{array}{l}\text { Non-odontogenic (benign) } \\
\text { tumor/tumor-like reactive lesions }\end{array}$} & \multirow[t]{2}{*}{ Male } & \multirow[t]{2}{*}{ Female } & \multicolumn{10}{|c|}{ Age distribution (years) } \\
\hline & & & $0-9$ & 10-19 & $20-29$ & $30-39$ & $40-49$ & $50-59$ & $60-69$ & $70-79$ & $80-89$ & $90-99$ \\
\hline \multicolumn{13}{|l|}{ Bone } \\
\hline Cemento-ossifying fibroma & 18 & 40 & 0 & 2 & 9 & 22 & 15 & 8 & 0 & 2 & 0 & 0 \\
\hline Cemental-osseous dysplasia & 2 & 33 & 0 & 4 & 4 & 3 & 9 & 10 & 4 & 1 & 0 & 0 \\
\hline Fibrous dysplasia & 11 & 18 & 0 & 2 & 12 & 8 & 4 & 3 & 0 & 0 & 0 & 0 \\
\hline Osteoma & 7 & 9 & 0 & 3 & 4 & 1 & 3 & 2 & 2 & 1 & 0 & 0 \\
\hline Central neurofibroma & 1 & 3 & 0 & 0 & 0 & 2 & 0 & 2 & 0 & 0 & 0 & 0 \\
\hline Osseous choristoma & 0 & 3 & 0 & 0 & 0 & 1 & 2 & 0 & 0 & 0 & 0 & 0 \\
\hline Central giant cell granuloma & 2 & 1 & 1 & 0 & 1 & 0 & 0 & 1 & 0 & 0 & 0 & 0 \\
\hline Osteoblastoma & 0 & 3 & 0 & 2 & 0 & 0 & 1 & 0 & 0 & 0 & 0 & 0 \\
\hline Central schwannoma & 1 & 2 & 0 & 0 & 0 & 0 & 2 & 1 & 0 & 0 & 0 & 0 \\
\hline Desmoplastic fibroma & 0 & 1 & 0 & 0 & 0 & 0 & 1 & 0 & 0 & 0 & 0 & 0 \\
\hline Osteolipoma & 0 & 1 & 0 & 0 & 0 & 0 & 0 & 0 & 0 & 1 & 0 & 0 \\
\hline Chondroid choristoma & 0 & 1 & 0 & 0 & 0 & 0 & 0 & 0 & 0 & 0 & 1 & 0 \\
\hline Ossifying fibromyxoid tumor & 0 & 1 & 0 & 0 & 0 & 0 & 1 & 0 & 0 & 0 & 0 & 0 \\
\hline Juvenile aggressive ossifying fibroma & 0 & 1 & 0 & 0 & 1 & 0 & 0 & 0 & 0 & 0 & 0 & 0 \\
\hline Synovial chondromatosis & 0 & 1 & 0 & 0 & 0 & 0 & 0 & 0 & 1 & 0 & 0 & 0 \\
\hline \multicolumn{13}{|l|}{ Salivary gland } \\
\hline Pleomorphic adenoma & 32 & 33 & 0 & 0 & 8 & 18 & 16 & 13 & 8 & 2 & 0 & 0 \\
\hline Warthin's tumor & 10 & 2 & 0 & 0 & 0 & 0 & 2 & 7 & 2 & 1 & 0 & 0 \\
\hline Papillary cystic adenoma & 4 & 4 & 0 & 0 & 3 & 0 & 0 & 2 & 0 & 3 & 0 & 0 \\
\hline Necrotizing sialometaplasia & 3 & 1 & 0 & 0 & 0 & 1 & 1 & 1 & 1 & 0 & 0 & 0 \\
\hline Adenomatoid hyperplasia & 2 & 0 & 0 & 0 & 1 & 0 & 0 & 1 & 0 & 0 & 0 & 0 \\
\hline Oncocytosis & 1 & 1 & 0 & 0 & 0 & 0 & 1 & 1 & 0 & 0 & 0 & 0 \\
\hline Tubular adenoma & 2 & 0 & 0 & 0 & 0 & 0 & 0 & 2 & 0 & 0 & 0 & 0 \\
\hline Benign lymphoepithelial lesion & 1 & 0 & 0 & 0 & 0 & 0 & 0 & 0 & 0 & 1 & 0 & 0 \\
\hline \multicolumn{13}{|l|}{ Epithelial } \\
\hline Hyperkeratosis* & 4294 & 485 & 4 & 29 & 252 & 938 & 1425 & 1444 & 438 & 216 & 31 & 2 \\
\hline Epithelial dysplasia* & 2648 & 251 & 0 & 0 & 60 & 374 & 765 & 1053 & 364 & 237 & 44 & 2 \\
\hline Oral submucous fibrosis* & 2330 & 170 & 0 & 11 & 171 & 534 & 741 & 701 & 247 & 93 & 2 & 0 \\
\hline Epithelial hyperplasia* & 2088 & 283 & 3 & 14 & 95 & 376 & 646 & 801 & 243 & 169 & 23 & 1 \\
\hline Verrucous hyperplasia* & 1726 & 124 & 1 & 2 & 33 & 308 & 572 & 599 & 157 & 169 & 8 & 1 \\
\hline Papilloma & 74 & 41 & 0 & 4 & 12 & 15 & 31 & 33 & 7 & 9 & 4 & 0 \\
\hline Seborrheic keratosis & 2 & 1 & 0 & 0 & 0 & 2 & 0 & 1 & 0 & 0 & 0 & 0 \\
\hline \multicolumn{13}{|l|}{ Soft tissue } \\
\hline Fibroma & 311 & 314 & 20 & 20 & 64 & 112 & 158 & 170 & 35 & 33 & 12 & 1 \\
\hline Hemangioma & 99 & 85 & 5 & 13 & 11 & 29 & 35 & 53 & 20 & 13 & 4 & 1 \\
\hline Fibrous hyperplasia & 98 & 83 & 0 & 3 & 5 & 8 & 16 & 19 & 50 & 54 & 26 & 0 \\
\hline Lipoma & 22 & 15 & 0 & 1 & 2 & 2 & 9 & 7 & 10 & 6 & 0 & 0 \\
\hline Verruca vulgaris & 14 & 21 & 0 & 1 & 5 & 8 & 12 & 9 & 0 & 0 & 0 & 0 \\
\hline Peripheral odontogenic fibroma & 7 & 22 & 0 & 1 & 5 & 7 & 10 & 6 & 0 & 0 & 0 & 0 \\
\hline Nevus & 11 & 14 & 1 & 1 & 8 & 7 & 4 & 3 & 1 & 0 & 0 & 0 \\
\hline Lymphangioma & 13 & 12 & 2 & 5 & 3 & 2 & 2 & 4 & 4 & 3 & 0 & 0 \\
\hline
\end{tabular}


Table 7 Number, sex and age distribution of patients presenting benign non-odontogenic tumor/tumor-like reactive lesions (Continued)

\begin{tabular}{|c|c|c|c|c|c|c|c|c|c|c|c|c|}
\hline Verruciform xanthoma & 17 & 3 & 0 & 0 & 1 & 3 & 4 & 8 & 2 & 2 & 0 & 0 \\
\hline Myxofibroma & 3 & 11 & 0 & 0 & 3 & 3 & 3 & 2 & 2 & 1 & 0 & 0 \\
\hline Schwannoma & 5 & 6 & 1 & 4 & 2 & 3 & 0 & 1 & 0 & 0 & 0 & 0 \\
\hline Neurofibroma & 7 & 5 & 0 & 0 & 2 & 0 & 5 & 4 & 1 & 0 & 0 & 0 \\
\hline Neuroma & 3 & 5 & 0 & 1 & 0 & 1 & 1 & 3 & 2 & 0 & 0 & 0 \\
\hline Xanthogranuloma & 2 & 5 & 0 & 0 & 1 & 1 & 0 & 5 & 0 & 0 & 0 & 0 \\
\hline Hemangioendothelioma & 5 & 0 & 0 & 0 & 1 & 1 & 1 & 2 & 0 & 0 & 0 & 0 \\
\hline Granular cell tumor & 2 & 3 & 0 & 0 & 3 & 1 & 1 & 0 & 0 & 0 & 0 & 0 \\
\hline Sclerosing hemangioma & 2 & 2 & 0 & 0 & 0 & 0 & 3 & 1 & 0 & 0 & 0 & 0 \\
\hline Peripheral giant cell granuloma & 3 & 0 & 0 & 0 & 0 & 0 & 1 & 1 & 0 & 0 & 1 & 0 \\
\hline Myofibroma & 0 & 2 & 0 & 0 & 0 & 1 & 0 & 1 & 0 & 0 & 0 & 0 \\
\hline Keratoacanthoma & 0 & 2 & 0 & 0 & 0 & 0 & 0 & 0 & 2 & 0 & 0 & 0 \\
\hline Peripheral odontogenic myxoma & 1 & 1 & 0 & 0 & 0 & 0 & 0 & 0 & 1 & 1 & 0 & 0 \\
\hline Peripheral ossifying fibroma & 0 & 2 & 0 & 0 & 0 & 0 & 1 & 1 & 0 & 0 & 0 & 0 \\
\hline Lipofibroma & 1 & 1 & 0 & 0 & 0 & 0 & 1 & 1 & 0 & 0 & 0 & 0 \\
\hline Oral focal mucinosis & 1 & 1 & 0 & 0 & 0 & 0 & 1 & 0 & 1 & 0 & 0 & 0 \\
\hline Neurofibromatosis & 1 & 0 & 0 & 0 & 0 & 0 & 0 & 1 & 0 & 0 & 0 & 0 \\
\hline Angiofibroma & 1 & 0 & 0 & 0 & 0 & 0 & 0 & 1 & 0 & 0 & 0 & 0 \\
\hline Fibrolipoma & 1 & 0 & 0 & 0 & 0 & 0 & 1 & 0 & 0 & 0 & 0 & 0 \\
\hline Leiomyoma & 1 & 0 & 0 & 0 & 0 & 0 & 0 & 1 & 0 & 0 & 0 & 0 \\
\hline Fibrous histiocytoma & 1 & 0 & 0 & 0 & 0 & 0 & 1 & 0 & 0 & 0 & 0 & 0 \\
\hline Angiomyxoma & 0 & 1 & 0 & 1 & 0 & 0 & 0 & 0 & 0 & 0 & 0 & 0 \\
\hline Lipoblastoma & 1 & 0 & 0 & 1 & 0 & 0 & 0 & 0 & 0 & 0 & 0 & 0 \\
\hline Solitary fibrous tumor & 0 & 1 & 0 & 0 & 0 & 1 & 0 & 0 & 0 & 0 & 0 & 0 \\
\hline Hemangiolymphangioma & 0 & 1 & 0 & 0 & 1 & 0 & 0 & 0 & 0 & 0 & 0 & 0 \\
\hline Total & 13892 & 2132 & 38 & 125 & 783 & 2793 & 4508 & 4990 & 1605 & 1018 & 156 & 8 \\
\hline
\end{tabular}

*Oral potentially malignant disorder.

dysplasia and fibrous dysplasia $(\mathrm{p}=0.4533)$. In cementoossifying fibroma, the percentage of males was significantly lower than females $(\mathrm{p}=0.0039)$. In cemento-osseous dysplasia, the percentage of males was significantly lower than females $(p<0.0001)$. In fibrous dysplasia, the percentage of males and females was similar $(\mathrm{p}=0.1936)$.

Pleomorphic adenoma was the most common lesion in the salivary gland subgroup compared with the other type $(\mathrm{p}<0.0001)$, with an almost equal sex distribution $(p=0.9013)$; this comprised about $67.0 \%$ of the lesions and was mostly noted in the fourth to the sixth decades of life. Warthin's tumor was the second most common lesion, in which the number of males was much higher than that of females $(p=0.0209)$.

The data of malignant non-odontogenic tumor/tumorlike reactive lesions are shown in Table 8 , with the number of male patients being much higher than that of female patients $(12: 1)(\mathrm{p}<0.0001)$; this was mostly located in the fifth and sixth decades of life compared with the other the age groups $(\mathrm{p}<0.0001)$. The highest number of lesions was noted in the epithelial subgroup, in which SCC predominated (82.0\%), followed by verrucous carcinoma (6.5\%). SCC had significant higher percentage than the other types $(\mathrm{p}<0.0001)$. The second highest number of lesions was found in the salivary gland subgroup, with mucoepidermoid carcinoma and adenoid cystic carcinoma, comprising about $77.0 \%$ of all lesions, being the two most common lesions. Mucoepidermoid carcinoma had significant higher percentage than the other types $(p<0.0001)$. Adenoid cystic carcinoma had higher percentage than the other types $(p=0.0009)$. On the other hand, the lowest number of lesions was found in the mesenchymal subgroup, the most frequent lesion being osteosarcoma, which comprised about $60.0 \%$ of the lesions. Osteosarcoma had higher percentage than malignant fibrous histiocytoma ( $\mathrm{p}=0.0348)$. The second lowest number of lesions was noted in the hematologic subgroup, in which non-Hodgkin's lymphoma and Langerhans cell 
Table 8 Number, sex and age distribution of patients presenting malignant non-odontogenic tumors

\begin{tabular}{|c|c|c|c|c|c|c|c|c|c|c|c|c|}
\hline \multirow{2}{*}{$\begin{array}{l}\text { Non-odontogenic (malignant) } \\
\text { tumors }\end{array}$} & \multirow[t]{2}{*}{ Male } & \multirow[t]{2}{*}{ Female } & \multicolumn{10}{|c|}{ Age distribution (years) } \\
\hline & & & $0-9$ & $10-19$ & $20-29$ & $30-39$ & $40-49$ & $50-59$ & $60-69$ & $70-79$ & $80-89$ & $90-99$ \\
\hline \multicolumn{13}{|l|}{ Mesenchymal } \\
\hline Osteosarcoma & 3 & 6 & 0 & 3 & 0 & 0 & 0 & 3 & 1 & 1 & 1 & 0 \\
\hline Malignant fibrous histiocytoma & 2 & 0 & 0 & 0 & 0 & 0 & 0 & 2 & 0 & 0 & 0 & 0 \\
\hline Malignant solitary fibrous tumor & 1 & 0 & 0 & 0 & 0 & 1 & 0 & 0 & 0 & 0 & 0 & 0 \\
\hline Neuroblastoma & 0 & 1 & 1 & 0 & 0 & 0 & 0 & 0 & 0 & 0 & 0 & 0 \\
\hline Ewing sarcoma & 1 & 0 & 0 & 1 & 0 & 0 & 0 & 0 & 0 & 0 & 0 & 0 \\
\hline Chondrosarcoma & 0 & 1 & 0 & 0 & 0 & 0 & 0 & 1 & 0 & 0 & 0 & 0 \\
\hline \multicolumn{13}{|l|}{ Hematologic } \\
\hline Non-Hodgkin's lymphoma & 13 & 6 & 0 & 1 & 3 & 0 & 5 & 5 & 0 & 4 & 1 & 0 \\
\hline Langerhans cell histocytosis & 12 & 0 & 0 & 0 & 2 & 6 & 2 & 2 & 0 & 0 & 0 & 0 \\
\hline Plasmacytoma & 3 & 4 & 0 & 0 & 0 & 0 & 5 & 2 & 0 & 0 & 0 & 0 \\
\hline Leukemia & 0 & 3 & 2 & 0 & 0 & 0 & 1 & 0 & 0 & 0 & 0 & 0 \\
\hline Hodgkin's lymphoma & 1 & 1 & 0 & 0 & 0 & 0 & 0 & 0 & 0 & 2 & 0 & 0 \\
\hline Malignant hemangioendothelioma & 0 & 1 & 0 & 0 & 1 & 0 & 0 & 0 & 0 & 0 & 0 & 0 \\
\hline Multiple myeloma & 0 & 1 & 0 & 0 & 0 & 0 & 0 & 0 & 0 & 1 & 0 & 0 \\
\hline \multicolumn{13}{|l|}{ Salivary gland } \\
\hline Mucoepidermoid carcinoma & 19 & 16 & 0 & 0 & 7 & 9 & 7 & 6 & 0 & 3 & 3 & 0 \\
\hline Adenoid cystic carcinoma & 18 & 10 & 0 & 0 & 1 & 4 & 3 & 5 & 0 & 12 & 3 & 0 \\
\hline Papillary cystic adenocarcinoma & 6 & 2 & 0 & 0 & 0 & 0 & 0 & 0 & 0 & 6 & 2 & 0 \\
\hline Adenocarcinoma, NOS & 4 & 3 & 0 & 0 & 2 & 0 & 2 & 3 & 0 & 0 & 0 & 0 \\
\hline Salivary duct carcinoma & 2 & 1 & 0 & 0 & 1 & 2 & 0 & 0 & 0 & 0 & 0 & 0 \\
\hline Acinic cell carcinoma & 1 & 0 & 0 & 0 & 0 & 0 & 0 & 0 & 0 & 1 & 0 & 0 \\
\hline \multicolumn{13}{|l|}{ Epithelial } \\
\hline Squamous cell carcinoma & 4628 & 332 & 0 & 0 & 49 & 603 & 1709 & 1735 & 439 & 350 & 71 & 4 \\
\hline Verrucous carcinoma & 359 & 34 & 0 & 1 & 0 & 42 & 113 & 136 & 40 & 52 & 9 & 0 \\
\hline Metastatic carcinoma & 319 & 29 & 0 & 0 & 0 & 46 & 137 & 128 & 22 & 14 & 1 & 0 \\
\hline Carcinoma in situ & 142 & 13 & 0 & 0 & 1 & 23 & 40 & 53 & 18 & 16 & 4 & 0 \\
\hline Undifferentiated carcinoma & 11 & 4 & 0 & 0 & 0 & 2 & 2 & 3 & 2 & 3 & 3 & 0 \\
\hline Spindle cell carcinoma & 10 & 1 & 0 & 0 & 0 & 4 & 1 & 4 & 0 & 2 & 0 & 0 \\
\hline Carcinosarcoma & 3 & 1 & 0 & 0 & 0 & 1 & 2 & 1 & 0 & 0 & 0 & 0 \\
\hline Total & 5558 & 470 & 3 & 6 & 67 & 743 & 2029 & 2089 & 522 & 467 & 98 & 4 \\
\hline
\end{tabular}

histocytosis, comprising about $81.5 \%$ of the lesions, were the two most frequent lesions.

The second greatest number of HN lesions was noted in the inflammatory/infective group (Table 9), which comprised about $31.8 \%$ of all the HN lesions, with a male to female ratio of about 1.7:1 ( $\mathrm{p}<0.0001)$. Candidiasis was the most common lesion in this group, followed by inflammation, with both lesions being located in the range of 50-59 years. In candidiasis, the 50-59 years had significant higher percentage than the other age groups $(\mathrm{p}<0.0001)$. In inflammation, the 50-59 years had significant higher percentage than the other age groups $(\mathrm{p}<0.0001)$, except for 40-49 age group ( $\mathrm{p}=0.0112)$. Apical granuloma, distributed evenly in the fourth to the sixth decades of age, was the third most common lesion in this group.

The data of the others/miscellaneous lesions are shown in Table 10, with the number of female patients being slightly higher than that of the male patients $(\mathrm{p}=0.8660)$. Exostosis, mostly distributed in the range of 50-59 years, was the most common lesion within this group and comprised about $44.0 \%$ of the lesions. The exostosis had the highest percentage than other types significantly $(\mathrm{p}<0.0001)$. Dense fibrotic dental follicle was the second most common lesion, which occurred mostly in the 
Table 9 Number, sex and age distribution of patients presenting inflammatory/infective lesions

\begin{tabular}{|c|c|c|c|c|c|c|c|c|c|c|c|c|}
\hline \multirow[t]{2}{*}{ Inflammatory/infective lesions } & \multirow[t]{2}{*}{ Male } & \multirow[t]{2}{*}{ Female } & \multicolumn{10}{|c|}{ Age distribution (years) } \\
\hline & & & $0-9$ & 10-19 & $20-29$ & $30-39$ & $40-49$ & $50-59$ & $60-69$ & $70-79$ & $80-89$ & $90-99$ \\
\hline Candidiasis & 1908 & 627 & 13 & 8 & 52 & 290 & 614 & 872 & 328 & 275 & 79 & 4 \\
\hline Inflammation & 1149 & 672 & 21 & 55 & 134 & 191 & 434 & 512 & 191 & 196 & 83 & 4 \\
\hline Apical granuloma & 551 & 844 & 0 & 49 & 242 & 312 & 340 & 320 & 55 & 66 & 10 & 1 \\
\hline Non-specific ulcer & 728 & 258 & 6 & 21 & 46 & 103 & 210 & 290 & 162 & 105 & 43 & 0 \\
\hline Mucocele & 539 & 428 & 103 & 179 & 320 & 161 & 91 & 66 & 25 & 19 & 3 & 0 \\
\hline Lymphadenitis & 539 & 43 & 1 & 1 & 14 & 86 & 228 & 179 & 62 & 9 & 2 & 0 \\
\hline Pyogenic granuloma & 323 & 189 & 8 & 13 & 44 & 93 & 128 & 134 & 43 & 40 & 8 & 1 \\
\hline Granulation tissue & 307 & 196 & 12 & 37 & 38 & 57 & 108 & 136 & 46 & 55 & 13 & 1 \\
\hline Lichen planus* & 201 & 268 & 0 & 5 & 28 & 59 & 121 & 177 & 45 & 30 & 4 & 0 \\
\hline Osteomyelitis & 141 & 145 & 0 & 3 & 10 & 19 & 45 & 76 & 39 & 64 & 25 & 5 \\
\hline Sequestrum & 149 & 113 & 1 & 2 & 6 & 20 & 39 & 72 & 44 & 50 & 25 & 3 \\
\hline Sialadenitis & 87 & 103 & 2 & 2 & 11 & 29 & 45 & 66 & 9 & 21 & 5 & 0 \\
\hline Scar tissue & 145 & 32 & 2 & 9 & 7 & 26 & 49 & 49 & 15 & 16 & 3 & 1 \\
\hline Inflammatory fibrous hyperplasia & 94 & 69 & 0 & 0 & 14 & 27 & 40 & 82 & 0 & 0 & 0 & 0 \\
\hline Apical scar & 63 & 69 & 0 & 2 & 26 & 36 & 36 & 32 & 0 & 0 & 0 & 0 \\
\hline Acanthosis & 89 & 12 & 0 & 2 & 5 & 30 & 27 & 37 & 0 & 0 & 0 & 0 \\
\hline Necrotic tissue & 72 & 18 & 3 & 2 & 4 & 4 & 24 & 35 & 10 & 6 & 2 & 0 \\
\hline Mucositis & 56 & 27 & 1 & 1 & 7 & 16 & 17 & 26 & 11 & 4 & 0 & 0 \\
\hline Osteoradionecrosis & 53 & 4 & 0 & 0 & 0 & 3 & 11 & 30 & 4 & 7 & 2 & 0 \\
\hline Fibrosis & 28 & 19 & 1 & 3 & 5 & 7 & 10 & 19 & 0 & 2 & 0 & 0 \\
\hline Foreign body granuloma & 33 & 13 & 0 & 0 & 0 & 6 & 19 & 18 & 0 & 1 & 2 & 0 \\
\hline Ranula & 20 & 18 & 0 & 2 & 16 & 6 & 3 & 11 & 0 & 0 & 0 & 0 \\
\hline Sinusitis & 22 & 11 & 0 & 5 & 5 & 4 & 8 & 8 & 2 & 1 & 0 & 0 \\
\hline Sialoithiasis & 20 & 9 & 0 & 0 & 8 & 2 & 4 & 10 & 1 & 4 & 0 & 0 \\
\hline Gingival hyperplasia & 14 & 10 & 2 & 3 & 8 & 6 & 1 & 0 & 1 & 3 & 0 & 0 \\
\hline Osteosclerosis & 9 & 14 & 0 & 1 & 7 & 3 & 5 & 7 & 0 & 0 & 0 & 0 \\
\hline Tuberculosis & 16 & 5 & 3 & 0 & 2 & 3 & 2 & 4 & 2 & 3 & 2 & 0 \\
\hline Epulis granulomatosum & 6 & 14 & 0 & 0 & 2 & 5 & 1 & 6 & 1 & 2 & 3 & 0 \\
\hline Pemphigus vulgaris* & 14 & 6 & 0 & 1 & 0 & 1 & 9 & 5 & 4 & 0 & 0 & 0 \\
\hline Actinomycosis & 9 & 8 & 0 & 2 & 0 & 2 & 2 & 5 & 1 & 5 & 0 & 0 \\
\hline Sjogren syndrome* & 1 & 14 & 0 & 1 & 1 & 2 & 4 & 3 & 1 & 3 & 0 & 0 \\
\hline Periodontitis & 8 & 6 & 0 & 1 & 1 & 1 & 5 & 3 & 1 & 2 & 0 & 0 \\
\hline Condensing osteitis & 7 & 6 & 0 & 0 & 0 & 0 & 2 & 5 & 4 & 2 & 0 & 0 \\
\hline Gingivitis & 7 & 5 & 0 & 0 & 0 & 3 & 2 & 5 & 2 & 0 & 0 & 0 \\
\hline Fistula & 10 & 2 & 0 & 1 & 0 & 1 & 6 & 2 & 1 & 1 & 0 & 0 \\
\hline Pseudoepitheliomatous hyperplasia & 6 & 2 & 0 & 0 & 0 & 0 & 2 & 6 & 0 & 0 & 0 & 0 \\
\hline Epulis fissuratum & 5 & 3 & 0 & 0 & 0 & 0 & 0 & 0 & 6 & 2 & 0 & 0 \\
\hline Periapical abscess & 6 & 1 & 0 & 0 & 0 & 0 & 0 & 6 & & 1 & 0 & 0 \\
\hline Glossitis & 0 & 6 & 0 & 0 & 0 & 1 & 0 & 3 & 0 & 2 & 0 & 0 \\
\hline Thrombus & 3 & 3 & 0 & 1 & 0 & 0 & 2 & 1 & 2 & 0 & 0 & 0 \\
\hline Polyp & 4 & 2 & 0 & 0 & 2 & 0 & 1 & 2 & 1 & 0 & 0 & 0 \\
\hline Pulp stone & 2 & 2 & 0 & 0 & 2 & 0 & 0 & 2 & 0 & 0 & 0 & 0 \\
\hline Pulpitis & 2 & 2 & 0 & 0 & 0 & 0 & 0 & 2 & 2 & 0 & 0 & 0 \\
\hline
\end{tabular}


Table 9 Number, sex and age distribution of patients presenting inflammatory/infective lesions (Continued)

\begin{tabular}{|c|c|c|c|c|c|c|c|c|c|c|c|c|}
\hline Plasma cell granuloma & 2 & 2 & 0 & 0 & 1 & 1 & 2 & 0 & 0 & 0 & 0 & 0 \\
\hline Amyloidosis & 3 & 0 & 0 & 0 & 0 & 0 & 0 & 1 & 1 & 0 & 1 & 0 \\
\hline Pemphigoid & 0 & 3 & 0 & 0 & 0 & 1 & 1 & 1 & 0 & 0 & 0 & 0 \\
\hline Paget's disease & 2 & 0 & 0 & 0 & 0 & 0 & 0 & 2 & 0 & 0 & 0 & 0 \\
\hline Histioplasmosis & 1 & 1 & 0 & 0 & 0 & 0 & 0 & 1 & 0 & 1 & 0 & 0 \\
\hline Herpes simplex infection & 2 & 0 & 0 & 0 & 0 & 0 & 0 & 2 & 0 & 0 & 0 & 0 \\
\hline Pericornitis & 2 & 0 & 0 & 0 & 0 & 0 & 0 & 2 & 0 & 0 & 0 & 0 \\
\hline Myositis & 1 & 0 & 1 & 0 & 0 & 0 & 0 & 0 & 0 & 0 & 0 & 0 \\
\hline Phlebolith with thrombosis & 0 & 1 & 0 & 0 & 0 & 0 & 0 & 0 & 1 & 0 & 0 & 0 \\
\hline Psoriasiform mucositis & 1 & 0 & 0 & 0 & 0 & 0 & 1 & 0 & 0 & 0 & 0 & 0 \\
\hline Acanthosis nigricans & 1 & 0 & 0 & 0 & 0 & 0 & 1 & 0 & 0 & 0 & 0 & 0 \\
\hline Tonsilitis & 0 & 1 & 0 & 0 & 0 & 1 & 0 & 0 & 0 & 0 & 0 & 0 \\
\hline Chondroid hyperplasia & 1 & 0 & 0 & 0 & 0 & 1 & 0 & 0 & 0 & 0 & 0 & 0 \\
\hline Nodular fascitis & 1 & 0 & 0 & 0 & 0 & 0 & 0 & 1 & 0 & 0 & 0 & 0 \\
\hline Socket sclerosis & 0 & 1 & 0 & 0 & 0 & 0 & 0 & 0 & 0 & 1 & 0 & 0 \\
\hline Caries & 1 & 0 & 0 & 0 & 0 & 0 & 0 & 0 & 0 & 1 & 0 & 0 \\
\hline Varicosities & 0 & 1 & 0 & 0 & 0 & 0 & 0 & 1 & 0 & 0 & 0 & 0 \\
\hline Lupus erythematosus & 1 & 0 & 0 & 0 & 0 & 1 & 0 & 0 & 0 & 0 & 0 & 0 \\
\hline Sialodochitis & 1 & 0 & 0 & 1 & 0 & 0 & 0 & 0 & 0 & 0 & 0 & 0 \\
\hline Phycomycosis & 0 & 1 & 0 & 1 & 0 & 0 & 0 & 0 & 0 & 0 & 0 & 0 \\
\hline Total & 7466 & 4309 & 180 & 414 & 1068 & 1620 & 2700 & 3335 & 1123 & 1000 & 315 & 20 \\
\hline
\end{tabular}

*Autoimmune disease.

first three decades of life. The dense fibrotic dental follicle had the highest percentage than the other types significantly $(\mathrm{p}<0.0001)$.

\section{Discussion}

The Oral Pathology Department not only provides services for nearly all the biopsied HN lesions (Medical Pathology contributes only a minor number of cases), but is also the most heavily used referral center for patients with these lesions in southern Taiwan. Therefore, the various types of lesions in the current study are representative of the occurrence of such lesions among the cohort of referral patients for a biopsy procedure in this geographical region.

In the current study, a total of 37,210 cases of HN lesions from 2000-2011 was documented in a cohort of Taiwanese patients referred for histopathological examination. SCC was the most common lesion in this cohort (13.3\%), which was in contrast to the findings of Franklin \& Jones [8] and Ali \& Sundaram [9], in which fibrous hyperplasia ( $14.7 \%$ and $20.7 \%$ respectively) was the most common lesion, as well as different to the findings of Bhasker [10] and Tay [11], in which dental granuloma (11.1\%) and fibrous epulis (10.3\%) were respectively the most frequent lesions. This may be due to the fact that betel quid chewing, which is a high risk factor for oral epithelial malignancies, has a high incidence in Taiwan [12]. This finding was consistent to the high incidence (16.2-39.0\%) of oral cancers in India [13], in which betel quid chewing has been responsible for half of oral cancer cases [14]. On the other hand, the prevalence of OSF, being one of the oral potential malignant disorders closely associated with betel quid chewing, of the present study $(6.7 \%)$ was compatible to that of the study from India (7.1\%) [15]. So, the high frequency of betel quid chewing in Taiwan may also contribute to the finding that five of the twelve most common lesions were potentially malignant disorders in the present study. However, it should be cautioned that the present cohort consisted only of patients and such patients were much more likely to be referred for histopathologcal assessments; hence, a higher prevalence of SCC and oral potentially malignant disorders would be expected to be observed.

Most lesions in the current study were distributed in the benign non-odontogenic tumor group (43.1\%), followed by the inflammatory/infective group (31.6\%), which was compatible with the findings of Jones \& Franklin [6]. On the other hand, the lowest numbers of lesions was in the non-odontogenic cyst/pseudocyst group, which was consistent with the other studies $[7,10,11]$. Moreover, oral epithelial malignant lesions comprised $16.2 \%$ of all 
Table 10 Number, sex and age distribution of patients presenting others/miscellaneous lesions

\begin{tabular}{|c|c|c|c|c|c|c|c|c|c|c|c|c|}
\hline \multirow{2}{*}{$\begin{array}{l}\text { Others/miscellanous } \\
\text { lesions }\end{array}$} & \multirow[t]{2}{*}{ Male } & \multirow[t]{2}{*}{ Female } & \multicolumn{10}{|c|}{ Age distribution (years) } \\
\hline & & & $0-9$ & 10-19 & $20-29$ & $30-39$ & $40-49$ & $50-59$ & $60-69$ & $70-79$ & $80-89$ & $90-99$ \\
\hline Exostosis & 119 & 142 & 1 & 4 & 9 & 41 & 56 & 99 & 14 & 34 & 3 & 0 \\
\hline Dense fibrotic dental follicle & 57 & 59 & 26 & 50 & 30 & 5 & 3 & 2 & 0 & 0 & 0 & 0 \\
\hline Foreign body & 34 & 15 & 0 & 2 & 3 & 3 & 18 & 15 & 5 & 3 & 0 & 0 \\
\hline Hematoma & 20 & 15 & 0 & 1 & 2 & 3 & 7 & 10 & 4 & 6 & 2 & 0 \\
\hline Melanotic macule & 8 & 17 & 0 & 0 & 5 & 6 & 3 & 9 & 0 & 1 & 1 & 0 \\
\hline Fordyce's granule & 12 & 6 & 0 & 1 & 1 & 2 & 10 & 3 & 1 & 0 & 0 & 0 \\
\hline Hypercementosis & 6 & 5 & 0 & 0 & 1 & 1 & 3 & 2 & 3 & 1 & 0 & 0 \\
\hline Supernumery tooth & 5 & 5 & 3 & 3 & 2 & 1 & 0 & 1 & 0 & 0 & 0 & 0 \\
\hline Blood clot & 6 & 3 & 0 & 1 & 1 & 0 & 1 & 1 & 1 & 2 & 2 & 0 \\
\hline Melanoplakia & 3 & 4 & 0 & 1 & 2 & 3 & 0 & 1 & 0 & 0 & 0 & 0 \\
\hline Internal root resorption & 1 & 3 & 0 & 2 & 1 & 0 & 0 & 0 & 0 & 1 & 0 & 0 \\
\hline Mesioden & 1 & 3 & 2 & 2 & 0 & 0 & 0 & 0 & 0 & 0 & 0 & 0 \\
\hline Calcification & 2 & 1 & 0 & 0 & 0 & 0 & 1 & 1 & 1 & 0 & 0 & 0 \\
\hline Osteoporosis & 2 & 0 & 0 & 0 & 0 & 0 & 0 & 0 & 0 & 2 & 0 & 0 \\
\hline Dentinogenesis imperfecta & 0 & 2 & 2 & 0 & 0 & 0 & 0 & 0 & 0 & 0 & 0 & 0 \\
\hline Melanosis & 1 & 1 & 2 & 0 & 0 & 0 & 0 & 0 & 0 & 0 & 0 & 0 \\
\hline Leukoedema & 1 & 0 & 0 & 0 & 0 & 1 & 0 & 0 & 0 & 0 & 0 & 0 \\
\hline Amalgum tatoo & 0 & 1 & 0 & 0 & 0 & 0 & 0 & 0 & 1 & 0 & 0 & 0 \\
\hline Epithelial atrophy & 1 & 0 & 0 & 0 & 0 & 0 & 0 & 0 & 0 & 1 & 0 & 0 \\
\hline Natal tooth & 0 & 1 & 1 & 0 & 0 & 0 & 0 & 0 & 0 & 0 & 0 & 0 \\
\hline Total & 279 & 283 & 37 & 67 & 57 & 66 & 102 & 144 & 30 & 51 & 8 & 0 \\
\hline
\end{tabular}

HN lesions in the present cohort, which was appeared to be much higher than the data of Bhaska (6.9\%) [10].

The overall male to female ratio $(3.4: 1)$ in the current study was higher than the studies of Ali (1.1:1) [7], Ali \& Sundaram (1.1:1) [9], Akinmoladun et al. (1.2:1) [5], and Jones \& Franklin (0.9:1) [6]. Most lesions were located in the range of 50-59 years, followed by 40-49 years, which may be attributed to the aging population becoming dominant in Taiwan [16]. Additionally, this finding was in contrast to the study of Akinmoladun et al. in Nigeria (range: 20-29 years) [5].

The lesions in the odontogenic cystic group were mostly distributed in the third to the sixth decade of life, and the number of males was slightly higher than the number of females; this result was compatible with the study of Avelar et al. [1]. Radicular cyst was the most common $(76.0 \%)$ of the lesions within the odontogenic cyst group in this study; this finding was similar to other studies [17-21]. Furthermore, most radicular cysts were distributed in the maxilla (64.7\%) in this cohort, which was similar to the results of Souza et al. (63.0\%) [21] and Prockt et al. (66.0\%) [20]. Dentigerous cyst was the second most common lesion (13.1\%), in which the ratio of males to females was about $2: 1$; this was similar to the study of Selvamani et al. [18]. More cases of dentigerous cyst were noted in Bhasker's study (33.8\%) [10] in comparison with the present study cohort. Additionally, although the majority of dentigerous cysts were located in the mandible $(62.4 \%)$ in this study, this was lower than the findings of Souza et al. (81.0\%) [21] and Prockt et al. (69.0\%) [20]. Keratocystic odontogenic tumor was the third most common lesion in this group (7.0\%), which was compatible with the study of Jones \& Franklin [6]; however, this lesion was the most frequent in the study of Koivisto et al. [22]. Moreover, most keratocystic odontogenic tumors were located in the mandible in this study cohort, similar to the results of the other studies $[18,19,21,22]$.

Epidermoid cyst was the most common lesion in the group of non-odontogenic cyst/pseudocyst in the current cohort, which was in contrast to the studies of Ali [7], Bhasker [10] and Tay [11], in which nasopalatine duct cyst was found to be the most common lesion respectively in these three studies. Lymphoepithelial cyst and nasopalatine duct cyst were the second and the third most common lesions in this group, but the numbers of cases of these two kinds of cyst were much lower than that of epidermoid cyst.

Odontoma was the most common lesion in the group of benign odontogenic tumor, constituting about $43.0 \%$ 
of the lesions, which was in contrast to the study of Ali [7]. The ratio of compound to complex odontomas in the current study (1.8:1) was compatible with the results of Bhaskar (2.1:1) [10] and Tay (2.4:1) [11] but in contrast to the data of Jones \& Franklin (1:1.6) [6] and Luo \& Li (1:1.4) [23]. Furthermore, most of the odontomas were diagnosed in the second decade of life in the study, which was the same as the result of Luo \& Li [23]. Although the majority of odontomas in the present study were located in maxilla (56.5\%), this was lower than the results of Servato et al. (66.6\%) [2] and Luo \& Li (66.0\%) [23]. Ameloblastoma was the second most common lesion in the study, with a male to female ratio of $1.37: 1$, which was compatible with the study of Siriwardena et al. (1.03:1) [24], who reported the average age to be 37.5 years, which was also compatible with the study (36.6 years). Additionally, similar to the other studies [2,24-26], most ameloblastomas in the present study were located in the mandible. Odontogenic fibroma was the third most common lesion in this group, which was different to the studies of Bhaskar [10] and Tay [11], who reported that it was the most common lesion. Most of the odontogenic fibromas in our cohort were diagnosed in the third decade of life, which was the same as the studies of Luo \& Li [23] and Servato et al. [2].

Pleomorphic adenoma (67.0\%) and Warthin's tumor $(12.5 \%)$ were the two most common lesions in the salivary gland subgroup, which was compatible with the results of Jaafari-Ashkavandi et al. (80.2\% and $10.5 \%$, respectively) [27] and Wang et al. (52.7\% and $17.4 \%$, respectively) [28]. Most pleomorphic adenomas in the present study were located in the palate $(60.0 \%)$, followed by the parotid gland $(30.0 \%)$, which was different to the studies of Jaafari-Ashkavandi et al. [27] and Wang et al. [28], in which most cases were located in the parotid gland (52.2\% and 52.7\%, respectively). Additionally, the male to female ratio of the cases of pleomorphic adenoma was almost 1:1 in the study, which was compatible with the report of Jaafari-Ashkavandi et al. (1.26:1) [27]. On the other hand, same as the study of Wang et al. [28], most Warthin's tumors in the present cohort were located in the parotid gland. The male to female ratio of Warthin's tumors in the cohort was 5:1, which was higher than the data of Wang et al. [28].

Fibroma was the most common lesion (69.4\%) in the soft tissue subgroup, which was compatible with the studies of Ali \& Sundaram [9] and Jones \& Franklin [6]. Most of the fibromas in the current study were found in the buccal mucosa; however, they most commonly occurred in the gingiva/alveolar ridge in the study of Ali \& Sundaram [9]. Hemangioma was the second most common lesion in the study, comprising cavernous (60.0\%) and capillary (40\%) types, which was compatible with the results of Jones \& Franklin $(62.0 \%$ and 38.0\%, respectively) [6]. Furthermore, most of the hemangiomas in the study were noted in the tongue, but no specific location has been documented in other studies [5-11].

In the current study, the number of female patients was about three times higher than that of male patients $(1: 3)$ in the bone subgroup, which was similar to the result of Ali (1:8) [7]. Cemento-ossifying fibroma was the most common lesion within this subgroup, which was in contrast to the results of Ali [7], who reported central giant cell granuloma to be the most common lesion. Most cemento-ossifying fibromas were located in the mandible, and were diagnosed most frequently in the fourth decade of life in the current study, which was the same as the data of MacDonald-Jankowski [29]. Cemento-osseous dysplasia was the second most common lesion, most documented in the fifth and sixth decades of life, and mostly in females; all these findings were compatible with the study of Alsufyani \& Lam [30]. Additionally, most of these lesions were located in the mandible $(83.0 \%)$, which was similar to the study of Alsufyani \& Lam (81.4\%) [30].

The lesions within the epithelial subgroup comprised about $39.0 \%$ of all $\mathrm{HN}$ lesions in the present cohort, which was much higher than the result of Amarasinghe et al. (11.3\%) in Sri Lanka [31]. Furthermore, the number of males was much greater than that of females in our study, which was compatible with the reports of Ali \& Sundaram [9] and Thomas et al. [32]; however, an almost equal sex distribution was reported in the study of Jones \& Franklin [6]. Worthy of note, five of the seven types of lesions in this subgroup were potentially malignant disorders, and were also categorized within the 12 most common lesions; this may be due to the high frequency of oral habits related to risk of oral malignancy in Taiwan. Most of these five potentially malignant disorders were documented in the fifth and sixth decades of life, and most were found in the buccal mucosa, which was compatible with the study of Thomas et al. [32]. HK was the most common lesion in the epithelial subgroup, followed by ED in the current study, which was compatible with the report of Jones \& Franklin [6].

Compatible with the study of Ali [7], the number of lesions in the bone subgroup in this study was low, osteosarcoma being the most common lesion, but only nine cases were documented. On the other hand, only $0.7 \%$ of lesions were noted in the hematologic subgroup, which was lower than the study of Jones \& Franklin [6].

Mucoepidermoid carcinoma and adenoid cystic carcinoma were the two most common malignant lesions in the salivary gland subgroup, which was similar to the results of Bradley \& McGurk [33] and Wang et al. [28] but in contrast with the findings of Jaafari-Ashkavandi et al. [27]. The male to female ratio (1.6:1) in this subgroup was compatible with the results of Zohreh et al. (1.2:1) [27] and Wang et al. (1.2:1) [28]. Most mucoepidermoid 
carcinomas were located in the hard palate and soft palate, which was different to other studies [27,28,33]. Additionally, the mean age at which mucoepidermoid carcinoma was diagnosed in our study (37.6 years) was compatible with the study of Wang et al. (39.5 years) [28]. On the other hand, most of the adenoid cystic carcinomas in our cohort were located in the submandibular gland, which was similar to the studies of Bradley et al. [33] and Wang et al. [28]. Moreover, the mean age (60.5 years) at which adenoid cystic carcinoma was diagnosed in the present study was seemed to be greater than the result of Wang et al. (46 years) [28].

SCC was the most common HN lesion (13.3\%) and also the most frequent lesion in the epithelial subgroup of malignant non-odontogenic tumors, which was appeared to be higher than the findings of Jones \& Franklin [6] and Tay (3.5\%) [11]. Most of the oral epithelial malignant lesions in the current study were located in the buccal mucosa, which was different to the results of Hernandez-Guerrero et al. [34] from Mexico, in which tongue cancer was the most commonly documented malignant lesion. The ratio of males to females in the cohort was 13.9:1, which was seemed to be much higher than the findings of the Internal Agency for Research On Cancer (3.3: 1) [35], Hernandez-Guerrero et al. (1.4: 1) [34] and Ferlay et al. [36]. Most of the SCCs in the study were distributed in the fifth and sixth decades of life, and the average age was 51.2 years; this result was largely compatible with the seventh and eighth decades of life reported in a North American population $[37,38]$. The proportion of SCC in patients less than 40 years of age was about $11 \%$ in this study, which was compatible with the finding in Indians [39]; moreover, the trend of patients of a younger age with oral cancer has also been documented in the studies from Scotland [40] and the UK [41].

Candidiasis comprised $6.8 \%$ of all $\mathrm{HN}$ lesions, which was appeared to be higher than the result of Jones \& Franklin (1.0\%) [6]. The male to female ratio of this lesion $(3: 1)$ in the cohort was seemed to be higher than that of Jones \& Franklin (1.69:1) [6]. Moreover, most cases of candidiasis were located in the buccal mucosa in the current study, followed by the tongue, which was the same as the study of Ali \& Sundaram [9]. Inflammation comprised $4.9 \%$ of all HN lesions, which was appeared to be greater than the studies of Tay (3.6\%) [11] and Jones \& Franklin (1.2\%) [6]. Apical granuloma comprised $3.7 \%$ of all lesions, which was lower than the results of Bhaskar (12.0\%) [10] and Tay (8.8\%) [11]. Most apical granulomas in the current cohort were located in the maxilla, but no specific location has been reported in other studies [5-11]. Exostosis and dense fibrotic dental follicles were the two most common lesions in the others/miscellaneous group, which mostly occurred in the maxilla and were distributed in the sixth and second decades of life.

\section{Conclusion}

The present study described in detail the frequency, age and sex distribution in a cohort of Taiwanese patients referred for histopathological examination. It showed trends similar to previous reports from other countries; however, some detailed information was different, perhaps due to the different criteria and different geographic distribution. Moreover, oral SCC and oral potentially malignant disorders comprised high percentages of all $\mathrm{HN}$ lesions in the cohort; nevertheless it should be cautioned that the sample being non-epidemiologic and would probably be greatly askew to a high rate of malignant/potentially malignant lesions when compared to an epidemiological sample.

\section{Abbreviations}

HN: Head \& neck; OMF: Oral and maxillofacial; SCC: Squamous cell carcinoma; HK: Hyperkeratosis; ED: Epithelial dysplasia; OSF: Oral submucous fibrosis; EH: Epithelial hyperplasia; ENT: Ear, nose \& throat.

\section{Competing interests}

The authors declare that they have no competing interests.

\section{Authors' contributions}

$\mathrm{FL}, \mathrm{KYH}$, and $\mathrm{YKC}$ are the primary writers of the manuscript and participated in the study implementing. YKC conceived of the study, and had made substantial contributions to conception and design, and revised the manuscript critically for important intellectual content. PHC implements all the required statistical analyses. WCW, LML, HCH, and JYC assisted in interpretation of data. CHC, and YKC are the principal investigators of clinical studies in this project. All authors read and approved the final manuscript.

\section{Acknowledgements}

We are grateful to health and welfare surcharge of tobacco products, the Ministry of Health and Welfare, Taiwan, Republic of China (MOHW103-TD-B111-05)

\section{Author details}

'Division of Periodontology, Kaohsiung Medical University Hospital, Kaohsiung, Taiwan. ${ }^{2}$ School of Dentistry, College of Dental Medicine, Kaohsiung Medical University, Kaohsiung, Taiwan. ${ }^{3}$ Division of Oral Pathology \& Maxillofacial Radiology, Kaohsiung Medical University Hospital, Kaohsiung, Taiwan. ${ }^{4}$ Oral \& Maxillofacial Imaging Center, College of Dental Medicine, Kaohsiung Medical University, Kaohsiung, Taiwan. ${ }^{5}$ Division of Oral \& Maxillofacial Surgery, Kaohsiung Medical University Hospital, Kaohsiung, Taiwan.

Received: 27 April 2014 Accepted: 17 July 2014

Published: 21 July 2014

\section{References}

1. Avelar RL, Antunes AA, Carvalho RW, Bezerra PG, Oliveira Neto PJ, Andrade ES: Odontogenic cysts: a clinicopathological study of 507 cases. J Oral Sci 2009, 51:581-586.

2. Servato JP, de Souza PE, Horta MC, Ribeiro DC, de Aguiar MC, de Faria PR, Cardoso SV, Loyola AM: Odontogenic tumours in children and adolescents: a collaborative study of 431 cases. Int J Oral Maxillofac Surg 2012, 41:768-773.

3. Zuniga MD, Mendez CR, Kauterich RR, Paniagua DC: Paediatric oral pathology in a Chilean population: a 15-year review. Int J Paediatr Dent 2013, 23:346-351. 
4. Correa L, Frigerio ML, Sousa SC, Novelli MD: Oral lesions in elderly population: a biopsy survey using 2250 histopathological records. Gerodontology 2006, 23:48-54.

5. Akinmoladun VI, Akintububo OB, Adisa AO, Ojo EO, Ayuba D: Evaluation of the histopathology of orofacial lesions in a North-East Nigerian tertiary centre. Ann Afr Med 2013, 12:105-109.

6. Jones AV, Franklin CD: An analysis of oral and maxillofacial pathology found in adults over a 30-year period. J Oral Pathol Med 2006, 35:392-401.

7. Ali MA: Biopsied jaw lesions in Kuwait: a six-year retrospective analysis. Med Princ Pract 2011, 20:550-555.

8. Franklin $C D$, Jones AV: A survey of oral and maxillofacial pathology specimens submitted by general dental practitioners over a 30-year period. Br Dent J 2006, 200:447-450.

9. Ali M, Sundaram D: Biopsied oral soft tissue lesions in Kuwait: a six-year retrospective analysis. Ann Afr Med 2012, 21:569-575.

10. Bhaskar SN: Oral pathology in the dental office: survey of 20,575 biopsy specimens. J Am Dent Assoc 1968, 76:761-766.

11. Tay $A B: A$ 5-year survey of oral biopsies in an oral surgical unit in Singapore: 1993-1997. Ann Acad Med Singapore 1999, 28:665-671.

12. Chung $\mathrm{CH}$, Yang $\mathrm{YH}$, Wang TY, Shieh TY, Warnakulasuriya S: Oral precancerous disorders associated with areca quid chewing, smoking, and alcohol drinking in southern Taiwan. J Oral Pathol Med 2005, 34:460-466.

13. Ramachandra NB: The hierarchy of oral cancer in India. Int J Head Neck Surg 2012, 3:143-146.

14. Travasso C: Betel quid chewing is responsible for half of oral cancer cases in India, finds study. BMJ 2013, 347:f7536.

15. Patil PB, Bathi R, Chaudhari S: Prevalence of oral mucosal lesions in dental patients with tobacco smoking, chewing, and mixed habits: A crosssectional study in South India. J Family Commun Med 2013, 20:130-135.

16. Yang $T$, Wang $C Y$, Jeng JH, Lin CP, Tsai YL: Geriatric oral health care in Taiwan: What do we need? J Dent Sci 2007, 2:59-64

17. Johnson NR, Savage NW, Kazoullis S, Batstone MD: A prospective epidemiological study for odontogenic and non-odontogenic lesions of the maxilla and mandible in Queensland. Oral Surg Oral Med Oral Pathol Oral Radiol 2013, 115:515-522.

18. Selvamani M, Donoghue M, Basandi PS: Analysis of 153 cases of odontogenic cysts in a South Indian sample population: a retrospective study over a decade. Braz Oral Res 2012, 26:330-334.

19. Sharifian MJ, Khalili M: Odontogenic cysts: a retrospective study of 1227 cases in an Iranian population from 1987 to 2007. J Oral Sci 2011 53:361-367.

20. Prockt AP, Schebela CR, Maito FD, Sant'Ana-Filho M, Rados PV: Odontogenic cysts: analysis of 680 cases in Brazil. Head Neck Pathol 2008, 2:150-156.

21. de Souza LB, Gordon-Nunez MA, Nonaka CF, de Medeiros MC, Torres TF, Emiliano GB: Odontogenic cysts: demographic profile in a Brazilian population over a 38-year period. Med Oral Patol Oral Cir Bucal 2010, 15:e583-e590.

22. Koivisto T, Bowles WR, Rohrer M: Frequency and distribution of radiolucent jaw lesions: a retrospective analysis of 9,723 cases. J Endod 2012, 38:729-732.

23. Luo HY, Li TJ: Odontogenic tumors: a study of 1309 cases in a Chinese population. Oral Oncol 2009, 45:706-711.

24. Siriwardena BS, Tennakoon TM, Tilakaratne WM: Relative frequency of odontogenic tumors in Sri Lanka: Analysis of 1677 cases. Pathol Res Pract 2012, 208:225-230.

25. Siar $\mathrm{CH}$, Lau SH, Ng KH: Ameloblastoma of the jaws: a retrospective analysis of 340 cases in a Malaysian population. J Oral \& Maxillofac Surg 2012, 70:608-615.

26. Saghravanian N, Jafarzadeh H, Bashardoost N, Pahlavan N, Shirinbak I: Odontogenic tumors in an Iranian population: a 30-year evaluation. J Oral Sci 2010, 52:391-396.

27. Jaafari-Ashkavandi Z, Ashraf MJ, Moshaverinia M: Salivary gland tumors: a clinicopathologic study of 366 cases in southern Iran. Asian Pac J Cancer Prev 2013, 14:27-30.

28. Wang $Y L$, Zhu $Y X$, Chen TZ, Wang $Y$, Sun GH, Zhang L, Huang $C P$, Wang $Z Y$, Shen Q, Li DS, Wu Y, Ji QH: Clinicopathologic study of 1176 salivary gland tumors in a Chinese population: experience of one cancer center 1997-2007. Acta Otolaryngol 2012, 132:879-886.
29. MacDonald-Jankowski DS: Ossifying fibroma: a systematic review. Dentomaxillofac Radiol 2009, 38:495-513.

30. Alsufyani NA, Lam EW: Osseous (cemento-osseous) dysplasia of the jaws: clinical and radiographic analysis. J Can Dent Assoc 2011, 77:b70.

31. Amarasinghe HK, Usgodaarachchi US, Johnson NW, Lalloo R, Warnakulasuriya S: Betel-quid chewing with or without tobacco is a major risk factor for oral potentially malignant disorders in Sri Lanka: a casecontrol study. Oral Oncol 2010, 46:297-301.

32. Thomas SJ, Harris R, Ness AR, Taulo J, Maclennan R, Howes N, Bain CJ: Betel quid not containing tobacco and oral leukoplakia: a report on a crosssectional study in Papua New Guinea and a meta-analysis of current evidence. Int J Cancer 2008, 123:1871-1876.

33. Bradley PJ, McGurk M: Incidence of salivary gland neoplasms in a defined UK population. Br J Oral \& Maxillofac Surg 2013, 51:399-403.

34. Hernandez-Guerrero JC, Jacinto-Aleman LF, Jimenez-Farfan MD, MacarioHernandez A, Hernandez-Flores F, Alcantara-Vazquez A: Prevalence trends of oral squamous cell carcinoma. Mexico City's General Hospital experience. Med Oral Patol Oral Cir Bucal 2013, 18:e306-e311.

35. Ferlay J, Shin HR, Bray F, Forman D, Mathers C, Parkin DM: Estimates of worldwide burden of cancer in 2008: GLOBOCAN 2008. Int J Cancer 2010, 127:2893-2917.

36. Ferlay J, Parkin DM, Steliarova-Foucher E: Estimates of cancer incidence and mortality in Europe in 2008. Eur J Cancer 2010, 46:765-781.

37. Chhetri DK, Rawnsley JD, Calcaterra TC: Carcinoma of the buccal mucosa. Otolaryngol Head Neck Surg 2000, 123:566-571.

38. Diaz EM Jr, Holsinger FC, Zuniga ER, Roberts DB, Sorensen DM: Squamous cell carcinoma of the buccal mucosa: one institution's experience with 119 previously untreated patients. Head Neck 2003, 25:267-273.

39. Elango JK, Gangadharan P, Sumithra S, Kuriakose MA: Trends of head and neck cancers in urban and rural India. Asian Pac J Cancer Prev 2006, 7:108-112.

40. Robinson KL, Macfarlane GJ: Oropharyngeal cancer incidence and mortality in Scotland: are rates still increasing? Oral Oncol 2003, 39:31-36.

41. Warnakulasuriya S, Mak V, Moller H: Oral cancer survival in young people in South East England. Oral Oncol 2007, 43:982-986.

doi:10.1186/1746-160X-10-28

Cite this article as: Lei et al:: Retrospective study of biopsied head and neck lesions in a cohort of referral Taiwanese patients. Head \& Face Medicine 2014 10:28.

\section{Submit your next manuscript to BioMed Central and take full advantage of:}

- Convenient online submission

- Thorough peer review

- No space constraints or color figure charges

- Immediate publication on acceptance

- Inclusion in PubMed, CAS, Scopus and Google Scholar

- Research which is freely available for redistribution 